\title{
La contratación pública responsable. Diseño de indicadores de medición
}

\author{
Herenia Gutiérrez Ponce \\ $M^{a}$ Teresa Nevado Gil \\ María Pache Durán
}

RESUMEN: Actualmente, la UE reconoce la importancia de la contratación pública como uno de los instrumentos que deben utilizarse para lograr un crecimiento inteligente, sostenible e integrador. En España, la Ley 9/2017, de 8 de noviembre, de Contratos del Sector Público, por la que se transponen al ordenamiento jurídico español las Directivas del Parlamento Europeo y del Consejo 2014/23/UE y 2014/24/UE, recientemente aprobada, plantea incorporar de forma transversal criterios socialmente responsables a la hora de preparar y ejecutar los contratos públicos, de tal forma que obliga a las empresas licitadoras a cumplir requisitos concretos sobre igualdad de género, condiciones laborales justas, consumo de comercio justo o eficiencia energética. Ante esta situación, las administraciones públicas han comenzado a elaborar guías y aprobar instrucciones para la implementación de cláusulas socialmente responsables en sus contrataciones. Sin embargo, a pesar de su importancia, no existe un instrumento de medida específico que evalúe este tipo de conductas. En este contexto, planteamos esta investigación con el objetivo de diseñar indicadores éticos y sostenibles, consistentes con la nueva Ley, que permitan medir y comparar relativamente los comportamientos responsables de las administraciones en relación a sus contrataciones públicas. Para lograr nuestro objetivo, planteamos un estudio exploratorio sobre la información disponible en las páginas web de los municipios españoles. Este instrumento podrá favorecer el enfoque de prácticas y políticas responsables en atención a las carencias observadas, contribuyendo a obtener no solo beneficios ambientales, sino también económicos y sociales, tanto para la Administración como para el sector privado y la sociedad.

PALABRAS CLAVE: Contratación Pública Sostenible, Indicadores, Responsabilidad Social, Cláusulas sociales y medioambientales.

CLAVES ECONLIT: A13, K12, K20, H57, H70, J58.

Cómo citar este artículo / How to cite this article: GUTIÉRREZ, H., NEVADO, Mª.T. \& PACHE, M. (2019): "La contratación pública responsable. Diseño de indicadores de medición", CIRIEC-España, Revista de Economía Pública, Social y Cooperativa, 96, 253-280. DOI: 10.7203/CIRIEC-E.96.12627.

Correspondencia: Herenia Gutiérrez Ponce, Universidad Autónoma de Madrid, herenia.gutierrez@uam.es; Ma

Teresa Nevado Gil, Universidad de Extremadura, tnevado@unex.es, y María Pache Durán, Universidad de

Extremadura, mpache@unex.es. 


\section{EXPANDED ABSTRACT}

\section{Responsible public procurement. Design of measurement indicators}

The European Union recognizes the importance of public procurement as one of the instruments that must be used to achieve smart, sustainable and inclusive growth. In Spain, Law 9/2017, of November 8, on Public Sector Contracts, transposes the European Parliament and Council Directives 2014/23 / EU and 2014/24 / EU into the Spanish legal system. This law aims to incorporate transversally socially responsible criteria when preparing and executing public contracts. In addition, it obliges bidding companies to meet specific requirements on gender equality, fair labor conditions, fair trade consumption or energy efficiency. Specifically, the basic objectives pursued by the Law are; the impartiality and objectivity of procedures, greater transparency in public procurement and improving the quality-price ratio. All this means guaranteeing the correctness of the procedures, avoiding any hint of corruption and using public procurement in a strategic manner to implement, with the resources that are managed through the contracts, public policies at communitarian and national levels in the social, environmental, and development fields and the innovation and promotion of the participation of small and medium enterprises in public procurement. Faced with this situation, public administrations have begun to develop guidelines and approve instructions for the implementation of socially responsible clauses in their hiring. However, despite its importance, there is no specific measurement instrument that evaluates this type of behavior.

In this context, we propose this research with the aim of facilitating a tool, composed of a series of ethical and sustainable indicators, in line with the new Law, which allow comparative measurement and comparison of the responsible behavior of administrations in relation to their public contracts. In this way, the contracting bodies must present in their specifications concrete measures to guarantee, on the one hand, integrity in their contracting procedures and, on the other hand, the strategic use of their contracts. All this it will allow to implement, with the resources that are managed through contracts, national and international policies in social, environmental, promotion of innovation and of the participation of small and medium enterprises in public procurement.

The research has revealed the existence of numerous guides and publications for the implementation of social, ethical and environmental clauses in public procurement, as well as instructions or agreements on sustainable public procurement. Specifically, in Spain, there are many administrations that, in recent years, have published good practice guides for the inclusion of Social Responsibility clauses in their contracts. In addition, it has been observed the continued existing commitment of the Spanish public administrations with the Social Responsibility, through the approval of Agreements or Instructions. Indeed, since 2005, in which the Good Governance Code of the General State Administration was approved in Spain, and in 2009, when the Spanish Federation of Municipalities and Provinces appro- 
ved its own Code of Good Governance, very significant steps have been taken in terms of Social Responsibility and sustainability. In the second stage of the study, a series of social and environmental indicators were created to evaluate and compare responsible practices in public procurement throughout the different phases of the contract (preparation, awarding, execution).

This first investigation has some limitations derived from the lack of quantitative data, however, like any exploratory study, it constitutes a hypothesis-generating element and serves as the basis for future investigations that allow evaluating the hiring of the entities that make up the public sector in relationship to social, environmental and ethical issues. At the same time, this proposal may favor focusing the practices and responsible policies to address the observed shortcomings, contributing to obtain economic and social environmental benefits, for the Administration and for the private sector and society in general.

According to the objectives formulated in this exploratory study, it has been proven that, increasingly and with a general nature, public administrations are aware and are concerned about the aspects that have to do with Transparency, Social Responsibility and sustainability. From the analysis of the current situation of sustainable public procurement, the important efforts of the initiatives, both national and international, aimed at strengthening a regulatory framework in this area are confirmed. Specifically, in Spain, it is observed that there are many administrations that adopt the commitment to be socially responsible in their hiring. However, the great task that remains to be done to adapt to the regulatory framework in these matters has been verified.

The importance of this work, because it is a pioneer, is that it has defined a series of indicators that serve to evaluate sustainability in public procurement. In addition, the proposed indicators allow to guide the implementation of good practices and can be used in future research as a basic methodological instrument to advance in the understanding and improvement of sustainable public procurement processes. All this will allow knowing the current situation of sustainable public procurement and being able to compare them over time, evaluate the impact of the approval of procurement laws in local administrations, determine good practices in public procurement, measure the effects of instructions or agreements on public procurement, identify if there are patterns of behavior among public administrations in relation to their responsible contracting and what could be the conditioning factors of sustainable practices in public procurement.

KEYWORDS: Sustainable Public Procurement, Indicators, Social Responsibility, Social and environmental clauses. 


\section{Introducción}

Las Administraciones Públicas (AAPP) juegan un papel significativo en el ejercicio de la Responsabilidad Social (RS) ${ }^{1}$. Por un lado, son actoras y, por otro, promotoras de conductas responsables. Según el Informe sobre actuación del Gobierno en el fomento de la $R S$ de las empresas en España (MTIN, 2009), existen cuatro aspectos que hacen que la Administración sea actora en el proceso de implantación de la RS: transparencia y gobernanza, empleabilidad socialmente responsable, consumo e inversión socialmente responsable (Nevado et al., 2013). De la misma forma, Subirats (2003) señalaba que la gestión pública, a diferencia de la privada, ha de permitir la expresión de unos valores que no son sólo instrumentales sino también políticos, es decir, no se ha de limitar a buscar la mejor manera de hacer las cosas, sino que ha de hacerlo buscando reconciliar una gran diversidad de intereses sociales antagónicos, conectando con los fines que justifican a la Administración Pública (AP). Debe orientarse a ofrecer un servicio de calidad a los ciudadanos, a cumplir sus necesidades y expectativas, buscando el equilibrio de intereses de todos los grupos que integran la sociedad, diseñando sus procesos para optimizar su función de servicio público. A esto alude, en definitiva, el concepto de gestión pública responsable (o de responsabilidad corporativa pública, utilizado por Cueto (2013)). En relación con estos dos últimos aspectos, las AAPP deben ser buenas inversoras y consumidoras, por lo que los criterios sociales y medioambientales que puedan requerir en sus adquisiciones, las compras públicas (contratos públicos), y en la forma de prestar sus servicios, son de especial relevancia, tanto en el aspecto interno como en provocar actuaciones externas. Se trata, por tanto, de integrar los enfoques de RS y de los principios del desarrollo sostenible en los sistemas de gestión pública para establecer requisitos, en términos socialmente responsables, de acuerdo con lo establecido en la legislación de contratos del sector público (Romodina y Silin, 2016). En este sentido, la RS en las AAPP supone un compromiso ético frente a los ciudadanos en el que también se incluyen compromisos sociales, económicos, laborales, medioambientales y de respeto a los derechos humanos en perfecta sintonía con todos los stakeholders (Gutiérrez-Ponce et al., 2018).

Por otro lado, las administraciones deben ser promotoras de comportamientos socialmente responsables a través de la creación de incentivos a las empresas y reformando la legislación. En España, en el ámbito de la Administración General del Estado, se han desarrollado iniciativas de promoción de conductas sostenibles, entre las que podemos mencionar la Ley de promoción de la autonomía personal y atención a personas en situación de dependencia², la Ley Orgánica para la igualdad efectiva

1.- Vamos a utilizar los términos Responsabilidad Social y Responsabilidad Social Corporativa (a partir de ahora RS o RSC) en un sentido amplio, en tanto responsabilidad de las organizaciones ante la sociedad o integración voluntaria de las preocupaciones sociales y medioambientales en sus actividades y en la relación con sus interlocutores.

2.- Ley 39/2006, de 14 de diciembre de dependencia de promoción de autonomía personal y atención a las personas en situación de dependencia (BOE DE 15/12/2006). 
de mujeres y hombres ${ }^{3}$, la Ley de Responsabilidad Medioambiental 4 , que transpone la Directiva Europea 2004/355, la Ley de Desarrollo Sostenible en el medio rural6, la Ley de Economía Sostenible ${ }^{7}$ que establece las medidas para la racionalización y contención del gasto, así como para incrementar la transparencia fiscal de las AAPP o la Ley 19/2013, de 9 de diciembre, de Transparencia, Acceso a la Información Pública y Buen Gobierno ${ }^{8}$, entre otras. Recientemente, la aprobación de la Ley 9/2017, de 8 de noviembre, de Contratos del Sector Público, por la que se transponen al ordenamiento jurídico español las Directivas del Parlamento Europeo y del Consejo 2014/23/UE y 2014/24/UE, de 26 de febrero de 2014, pone de manifiesto el compromiso del Estado con la sostenibilidad a través de la inclusión de criterios sociales, ambientales y éticos en sus contrataciones.

Muchos autores, no obstante, se están mostrando críticos con esta "moda regulatoria" y, en concreto, los códigos de conducta por su papel más bien modesto en cuanto a resultados conseguidos (Villoria, 2006) y su carácter declarativo, cuando no meramente cosmético, al no estar integrados en un marco de integridad institucional con sistemas de seguimiento y control de su incumplimiento (Jiménez, 2013). En este sentido, es importante evaluar el grado de cumplimiento de esta nueva Ley en cuanto a si la contratación pública está teniendo en cuenta en las cláusulas de contratación estos criterios socialmente responsables. Son muchos los acuerdos, guías y publicaciones que han ido desarrollando distintas AAPP, que incorporan ejemplos y buenas prácticas como medidas ejemplares para el impulso de la contratación pública sostenible (García, 2016; Vañó, 2016). Sin embargo, a pesar de su importancia, no existe un instrumento de medida específico que evalúe este tipo de conductas.

Tal y como señalan Gutiérrez-Ponce et al. (2018), se hace necesario, en las AAPP en general y en las locales en particular, desarrollar una cultura de la RS que conlleve la prestación de los servicios sociales y al mismo tiempo, la sostenibilidad de los mismos, de tal manera que, las variables de eficiencia y control del gasto, acompañadas de un fortalecimiento financiero, permitan la sostenibilidad de los servicios que prestan los municipios y administraciones locales. Un aspecto menos desarrollado, como señala Melle (2007), es el que tiene que ver con las memorias RS, o la información que la organización debe proporcionar para hacerlas verificables y transparentes, asegurando su calidad y verificación externa e independiente, que es lo que permitiría evaluar la auténtica "convicción RS" de la organización, o la asunción de los auténticos valores que trata de promover la filosofía de la RS.

Por todo ello, el objetivo de este trabajo consiste en facilitar una herramienta que permita medir y comparar relativamente los comportamientos responsables de las administraciones en relación a sus contrataciones públicas. Para alcanzar nuestro objetivo, se ha llevado a cabo un estudio exploratorio

3.- Ley Orgánica 3/2007, de 22 de marzo para la igualdad efectiva de mujeres y hombres (BOE de 23/03/2007).

4.- Ley 26/2007, de 23 de octubre, de Responsabilidad Medioambiental (BOE de 24/10/2007).

5.- Más información en: http://eur-lex.europa.eu/LexUriServ/LexUriServ.do?uri=OJ:L:2004:143:0056:0075:es:PDF. Consultada a fecha 14/04/2018.

6.- Ley 45/2007, de 13 de diciembre para el Desarrollo Sostenible del medio rural (BOE de 14/12/2007).

7.- Ley 2/2011, de 4 de marzo, de Economía Sostenible (BOE de 05/03/2011).

8.- Ley 19/2013, de 9 de diciembre, de Transparencia, Acceso a la Información Pública y Buen Gobierno (BOE de 13/12/2013). 
sobre la información relativa a contratación pública sostenible que los municipios españoles suministran a través de sus páginas web. Posteriormente, con la información recopilada (guías y publicaciones para la implementación de cláusulas sociales, éticas y medioambientales en las contrataciones públicas, así como instrucciones o acuerdos sobre contratación pública sostenible), se ha confeccionado una relación de indicadores construida en consonancia con la Ley 9/2017, de 8 de noviembre, de Contratos del Sector Público, por la que se transponen al ordenamiento jurídico español las Directivas del Parlamento Europeo y del Consejo 2014/23/UE y 2014/24/UE, de 26 de febrero de 2014.

Este instrumento permitirá evaluar las contrataciones de las entidades que conforman el sector público en relación a cuestiones sociales, ambientales y éticas. Por otro lado, puede favorecer a enfocar las prácticas y políticas responsables en atención a las carencias observadas, contribuyendo a obtener, no solo beneficios ambientales, sino también económicos y sociales, tanto para la Administración como para el sector privado y la sociedad.

El trabajo se estructura como sigue. Tras la presente introducción, las siguientes secciones analizan la gestión y la contratación pública responsable. Seguidamente, se explican los objetivos y la metodología utilizada para la elaboración de la propuesta de medida que se presenta. Por último, se comentan las conclusiones del estudio.

\section{Aproximación al concepto de gestión pública responsable y sostenible}

Con la expresión Gestión Pública Responsable (GPR) aludimos al fundamento del comportamiento de las organizaciones públicas, al conjunto de valores y principios que motivan sus acciones y a una nueva cultura sobre gestión pública, entendida como la parte de la ética de las organizaciones públicas que incorpora el diálogo, la participación de todos los grupos de interés y la rendición de cuentas. Para Brugué (2004) la chispa transformadora de la AP está en el conjunto de mutaciones sociales de fondo producidas, que han dejado el modelo tradicional de Administración en una situación muy delicada y que requiere inventar algo diferente para responder a las exigencias sustantivas de un nuevo orden social, partiendo del reconocimiento de las dificultades de esa Administración para dar respuesta a las exigencias de una sociedad más compleja, más inestable y más diversa. Por tanto, la clave es la responsabilidad, no el control o la economía como se plantea en la Administración burocrática.

En este sentido, la RS en la Administración conecta, así, con valores éticos como responsabilidad, integridad, transparencia, objetividad, profesionalidad, o imparcialidad, y coloca al ciudadano como centro de su actividad, el que da sentido a su existencia (Gelderman et al., 2017). Esto significa que la ética es referencia necesaria para orientar las decisiones en el ámbito de la gestión pública: 
Los valores sirven de guía acerca de lo que es bueno al interés público. Como señala Canales (2003), "La ética pública es la seña identificadora de la función pública, que se caracteriza por el servicio público o, más gratificante, por el servicio al público, que son dos manifestaciones de la misma disposición general, en la medida en que se orienta a la consecución del bien común".

Para Melle (2007), a los principios tradicionales de actuación de la Administración (transparencia, disciplina presupuestaria, independencia, rendición de cuentas e imparcialidad) se debe añadir la RS para poder hablar de una auténtica GPR. Esta autora señala los siguientes principios generales para una gestión responsable en la AP: 1 . Identificación objetiva de las prácticas sostenibles y de todas las partes interesadas y/o afectadas (stakeholders), a través de un diagnóstico RS; 2. Incorporación de la RS en la gestión y en los procedimientos, involucrando a todas las partes interesadas y consiguiendo el compromiso de los puestos directivos; 3 . Adopción de códigos formales de RS, con políticas al respecto y articulación del diálogo con todos los stakeholders; 4. Compromiso ético, respeto hacia la diversidad e igualdad de oportunidades, con un reconocimiento explícito del valor positivo de la diversidad, y 5. Memorias RS con verificación externa.

La noción de GPR (Trujillo et al., 2011) nace ligada a dos conceptos próximos: por un lado, el de RS asociado a la gestión empresarial y, por otro, al de Buen Gobierno de las AAPP, como compromiso de cumplir con los derechos humanos, laborales y medioambientales y con la búsqueda de una gestión pública más transparente. Para perfilar el concepto de GPR se hace necesario precisar primero algunos aspectos relacionados con la responsabilidad y la AP, comenzando por el propio término Administración.

Actualmente, AP es un término con límites imprecisos que hace referencia al conjunto de las organizaciones que realizan la función administrativa del Estado y en cuya definición Sánchez (2001) incluye los siguientes elementos: 1. Tiene como finalidad la satisfacción de las necesidades públicas y la contribución a alcanzar los fines del Estado. 2. Comienza donde termina la política y termina donde comienza la política 3. Es un instrumento del Estado para realizar aquellas funciones que el mercado no hace y que, por su interés público, deben realizarse 4. Pone en contacto a los ciudadanos con el poder político 5 . Su razón de ser son los servicios que presta a la sociedad, esto es, atender a los intereses y derechos de los ciudadanos y grupos que forman la sociedad, buscando el equilibrio de todos ellos.

De aquí se deriva otro concepto fundamental para entender la Administración de los intereses generales y del bien común: el concepto de responsabilidad, que alude a rendir cuentas de los propios actos, cumplir con el deber o, también, poner cuidado en lo que se hace. La responsabilidad es un elemento central en una sociedad democrática, y el mejor antídoto contra la desconfianza de los ciudadanos en las instituciones y en lo público. La propia idea de lo que es justo o no incluye en sí misma la búsqueda de una responsabilidad y, si a todas las organizaciones sociales se las vigila y pide que sean responsables, más aún se debe hacer con la AP. Se trata de un elemento fundamental es las democracias modernas. 
Un segundo concepto a perfilar es el de RS en cuanto plus de comportamiento que va más allá de lo exigido por las normas legales, y que alude a la responsabilidad ética de las organizaciones. Tal como la define el Libro Verde de la Comisión Europea (COM, 2001), la RS significa, no sólo cumplir plenamente con las obligaciones jurídicas, sino concebir la actividad de la organización como una tarea de responsabilidad frente a todos los grupos e individuos involucrados $\mathrm{y} / 0$ afectados por sus actividades 0 sus decisiones, e incluir en su estrategia las preocupaciones sociales, laborales, medioambientales y de respeto a los derechos humanos en el diálogo con todos sus stakeholders.

En definitiva, es una nueva cultura y forma de pensar de las organizaciones, ya sean públicas 0 privadas, como parte de la ética o carácter positivo por el cual asumen en forma voluntaria una orientación estratégica que tiene en cuenta los intereses de todos sus grupos de interés.

La pluralidad de valores de las sociedades actuales y la consiguiente diversificación de las demandas requieren de la nueva Administración una reflexión profunda sobre la emergencia de un nuevo paradigma sobre el que construir el papel del Estado, y la relación de éste con la ciudadanía (Aldeguer, 2013). Desarrollar un nuevo marco de referencia o una nueva cultura y un nuevo modelo de gestión pública apropiados al momento actual y construir AAPP profesionales, capaces de asegurar la igualdad de todos en el acceso al ejercicio de sus derechos y a relacionarse con la Administración, removiendo los obstáculos que lo dificultan y adaptándose a la complejidad de las agendas públicas de hoy.

El desafío pasa por la transformación de las organizaciones públicas siguiendo un modelo acorde con la era de la información, y el concepto de innovación debe incluir tanto la innovación tecnológica (equipamientos, técnicas y sistemas) como organizativa (estructuras, estrategias, procedimientos administrativos y sistemas de gestión). Hablamos de una Administración electrónica, o de la incorporación de las tecnologías de la información para proporcionar y mejorar los servicios y transacciones que tiene la AP con los ciudadanos y con otras instituciones, actuando en sus dos vertientes: una intraorganizativa (transformando la oficina tradicional, sustituyendo los procesos en papel por procesos electrónicos) y otra externa (habilitación de la vía electrónica como medio preferente para la relación con los ciudadanos y empresas).

Hoy no es posible hablar de eficiencia ni de innovación sin contar con la simplificación de procesos que posibilitan las tecnologías de la información y la comunicación y la eliminación de los procesos de intermediación que no aportan valor. Pero la e-administración no debe ser vista sólo como una herramienta de mejora administrativa por la implantación de aplicaciones basadas en Internet 0 de facilitación del acceso de los ciudadanos a la Administración, sino que, como señala Subirats (2012) tiene que servir para repensar y redefinir los servicios en sí mismos. 


\section{La regulación de la $\mathrm{RS}$ y el papel de la administración pública}

Los estados y los organismos internacionales vienen haciendo, desde hace décadas, un esfuerzo regulatorio y promotor importante para que las empresas adopten criterios RS en sus actuaciones. Organismos como la Unión Europea, la ONU o la OCDE han realizado importantes esfuerzos por trasladar el debate internacional de la RS a los contextos nacionales y locales. La Unión Europea promueve la puesta en práctica de políticas de RS desde la Cumbre Europea de Lisboa (2000) y la Agenda Social de la Cumbre de Niza (2000), dándole forma en el Libro Verde de la RSE (2001) que surge como una llamada de la Comisión a las empresas para que participen en la lucha contra la exclusión. Parte de la consideración de que están en unos entornos cada vez más complejos donde, a su actividad tradicional, deben añadir otras variables relacionadas con las nuevas inquietudes y exigencias de sus grupos de interés y de la sociedad, el respeto a criterios sociales y medioambientales en sus decisiones de compra, la preocupación por el medio ambiente, o la transparencia en sus actividades.

El Libro Verde delimita las dos dimensiones que posee la RS: una interna (hacia el interior de la empresa) y otra externa (referida a sus interlocutores externos 0 stakeholders), y realiza una definición en la que se enfatiza que la RS significa, además del cumplimiento estricto de las obligaciones legales, un plus voluntario integrado en la estructura de la empresa en relación con las preocupaciones sociales, laborales, medioambientales y de respeto a los derechos humanos, responsabilizándose de todos los impactos que se deriven de sus acciones.

Posteriormente, a través de directivas y con el desarrollo de la Agenda Social Europea, ha ido orientando la acción de los gobiernos de la UE en este terreno. Por su parte, las Naciones Unidas aprueban en la Cumbre de la Tierra de Río de Janeiro (1992) la Agenda 21, como programa amplio con un conjunto de estrategias dirigidas a detener e invertir los efectos de la degradación del medio ambiente, buscando un desarrollo sostenible. La Agenda 21 supone un paso hacia una auténtica filosofía del desarrollo sostenible, transformando el modelo de desarrollo por uno nuevo que satisfaga las necesidades actuales de los ciudadanos sin poner en riesgo a las generaciones futuras.

En paralelo, en estos mismos años se adapta la normativa española, siguiendo directivas europeas, en aspectos relacionados con la eficiencia energética de los edificios, la contratación pública, o la accesibilidad y no discriminación de las personas con discapacidad en el acceso a los edificios y servicios públicos. También a nivel autonómico se han producido iniciativas de estímulo a la RS, siguiendo la línea de lo que hemos visto a nivel de la Administración General del Estado. 
Merece la pena destacar, por el grado de detalle al que desciende, la Ley de RSE aprobada por el Parlamento de Extremadura en 2010. La AP extremeña asume con esta ley una óptica de RS en su calidad de: a) consumidora: plan de equipamiento, eliminación progresiva del papel, adquisición de productos ecoetiquetados, eficiencia energética, gestión de residuos; b) inversora: plan de ahorro y eficiencia energética en la contratación pública de obra nueva o de reforma, adquisición de equipamiento para sus edificios administrativos (incluido el ofimático); c) contratante: inclusión de cláusulas sociales y medioambientales en los pliegos de contratación; d) empleadora: conciliación de la vida laboral y familiar, promoción de la igualdad, contratación de personas en riesgo de exclusión; y e) prestadora de servicios: simplificación documental, tramitación automatizada de los procedimientos, sede electrónica accesible, accesibilidad de las instalaciones.

Además, en sus relaciones con terceros una Administración responsable debe realizar políticas públicas que incorporen incentivos a las organizaciones que desarrollen un comportamiento socialmente responsable, vinculando el gasto público a las empresas socialmente responsables (Oruezabala y Rico, 2012; Burzaco, 2016). Su volumen de contratación de bienes y servicios le permite estimular a aquellas empresas que produzcan bienes o servicios más sostenibles y responsables a través de las políticas de contratación y de compra responsables, tanto en la selección del producto como del proveedor (compra pública responsable) (Shadrina y Romodina, 2017).

La Comisión Europea, en el año 2008, definió la contratación pública responsable como "un proceso mediante el cual los poderes públicos tratan de adquirir bienes, servicios y obras con un impacto ambiental reducido durante todo su ciclo de vida y unas consideraciones sociales y éticas, en comparación con los bienes, servicios y obras con la misma función primordial que de otra manera se adquirirían" (COM, 2008).

Más adelante, en el año 2011, se refiere a ella como "operaciones de contratación que tienen en cuenta uno o más de los siguientes aspectos sociales: oportunidades de empleo, trabajo digno, cumplimiento con los derechos sociales y laborales, inclusión social (incluidas las personas con discapacidad), igualdad de oportunidades, diseño de accesibilidad para todos, consideración de los criterios de sostenibilidad, incluidas las cuestiones de comercio ético, y un cumplimiento voluntario más amplio de RS de las empresas, a la vez que se respetan los principios consagrados en el Tratado de la Unión Europea y las Directivas de contratación" (COM, 2011). Según esto, a través de la contratación pública, los poderes públicos pueden realizar una política de intervención en la vida económica, social y política del país, que permita conseguir objetivos sociales, ambientales o de investigación (Brammer y Walker, 2011).

Las AAPP son organizaciones con un papel importante de RS en su propia gestión. En cuanto organizaciones que prestan servicios a los ciudadanos, deben avanzar en la implantación de prácticas de gestión responsable -predicar con el ejemplo-y no aconsejar a los demás lo que deben hacer, sin empezar por ellas mismas, porque pierden toda legitimidad ante la sociedad. Como se recoge en la Recomendación 34 del Informe de la Subcomisión del Congreso: "La Administración del Estado, de 
las Comunidades Autónomas, las Empresas Públicas y la Administración Local deben ser ejemplares en la integración de los principios y prácticas de la RS en su propio funcionamiento".

Con la entrada en vigor, en España, el pasado 9 de marzo, de la Ley 9/2017, de 8 de noviembre, de Contratos del Sector Público, por la que se transponen al ordenamiento jurídico español las Directivas del Parlamento Europeo y del Consejo 2014/23/UE y 2014/24/UE, de 26 de febrero de 2014, se profundiza en la vertiente social y medioambiental de la contratación pública. Los objetivos principales de esta Ley son, por un lado, garantizar la transparencia en todos los contratos y en todas sus fases, y, por otro, utilizar estratégicamente la contratación para coadyuvar a las políticas públicas en materia social, medioambiental, de innovación y de fomento de la contratación con pequeñas y medianas empresas.

Concretamente, los objetivos básicos que persigue la Ley y proclama desde su exposición de motivos son la integridad y estrategia, lograr una mayor transparencia en la contratación pública, y conseguir una mejor relación calidad-precio. Ello supone garantizar la integridad de los procedimientos, intentando atajar todo atisbo de corrupción, y utilizar la contratación pública de manera estratégica, para implementar con los recursos que se gestionan mediante contratos, las políticas públicas comunitarias y nacionales en materia social, medioambiental, de fomento de la innovación y de promoción de la participación de la pequeña y mediana empresa en la contratación pública.

Por lo tanto, de la revisión de la literatura y de la legislación más relevante sobre transparencia y RS pública hemos identificado la necesidad de llevar a cabo estudios que ayuden a definir un instrumento de medida específico que evalúe este tipo de conductas. Por ello, el objetivo de este trabajo consiste en facilitar una herramienta, compuesta por una serie de indicadores éticos y sostenibles, en consonancia con la nueva Ley, que permitan medir y comparar relativamente los comportamientos responsables de las administraciones en relación a sus contrataciones públicas. En este sentido, los órganos de contratación deben concretar en sus pliegos de condiciones medidas específicas para garantizar, por un lado, la integridad en sus procedimientos de contratación y, por otro, la utilización estratégica de sus contratos para implementar, con los recursos que se gestionan mediante contratos, las políticas nacionales e internacionales en materia social, medioambiental, de promoción de la innovación y de fomento de la participación de las pequeñas y medianas empresas en la contratación pública. Por eso, de acuerdo al objetivo enunciado, nos hemos planteado las siguientes preguntas de investigación:

- Q1. ¿Cuál es la situación actual de las contrataciones públicas sostenibles en España?

- Q2. ¿Cuál ha sido el impacto de la aprobación de las leyes de contratación en las administraciones locales?

- Q3. ¿Cuál es el impacto de las buenas prácticas en la contratación pública?

- Q4. ¿Cuáles son los efectos de las instrucciones o acuerdos sobre contratación pública sostenible en España? 
- Q5. ¿Cuáles son los principios de la RS y de sostenibilidad centrados en la iniciativa de las AAPP y su impacto en los municipios españoles?

- Q6. ¿Existen patrones comunes de comportamiento entre las AAPP en relación a sus contrataciones públicas responsables?

- Q7. ¿Cuáles son los factores que pueden influenciar las prácticas sostenibles en las contrataciones públicas?

\section{Metodología}

Para alcanzar los objetivos propuestos, planteamos un estudio exploratorio, descriptivo y analítico sobre la contratación pública sostenible a partir de la observación directa de la información contenida en las páginas web de los, aproximadamente, 9.500 municipios y otros entes locales españoles. Como todo estudio exploratorio "sirve para aumentar el grado de familiaridad con fenómenos relativamente desconocidos, obtener información sobre la posibilidad de llevar a cabo una investigación más completa" (Hernández et al., 2003).

La primera etapa del estudio, llevada a cabo durante los meses de noviembre de 2017 hasta febrero de 2018, ha consistido en el análisis de las webs de las entidades locales españolas recopilando información sobre contratación pública y directrices para la inclusión de criterios sostenibles en los procesos de contratación pública de diversas AAPP españolas. Además, de detectar algún instrumento de medida específico que evalúe este tipo de conductas.

En la segunda etapa de nuestra investigación se ha realizado un análisis minucioso de la información contenida en estas iniciativas y experiencias municipales. Por último, tras un esfuerzo de sistematización y de síntesis, se ha diseñado una herramienta, consistente con los pronunciamientos internacionales generalmente aceptados, en consonancia con la Ley 9/2017, de 8 de noviembre, de Contratos del Sector Público, por la que se transponen al ordenamiento jurídico español las Directivas del Parlamento Europeo y del Consejo 2014/23/UE y 2014/24/UE, de 26 de febrero de 2014. Además, para la elaboración de la herramienta de medición, se ha tenido en cuenta lo establecido en la citada Ley, en relación a la inclusión de los criterios sociales y medioambientales en las distintas fases del contrato: 


\section{Fase de preparación del contrato}

\section{Objeto del contrato}

El momento de definir el objeto del contrato es la primera ocasión en la que los órganos de contratación tendrán la oportunidad de incluir criterios sociales y medioambientales en la licitación de contratos públicos. Esta es la fase más importante puesto que permitirá la incorporación posterior de dichos criterios en las siguientes fases, ya sea en los criterios de adjudicación como en las condiciones especiales de la ejecución del contrato que se establecen en el Pliego de Cláusulas Administrativas.

\section{Prohibición para contratar}

El Artículo 71.1.d de la Ley 9/2017, de 8 de noviembre, de Contratos del Sector Público, por la que se transponen al ordenamiento jurídico español las Directivas del Parlamento Europeo y del Consejo 2014/23/UE y 2014/24/UE, de 26 de febrero de 2014, penaliza a las empresas que vulneren alguna normativa social o medioambiental, estableciendo como causa de prohibición de contratar con las administraciones: (1) En el ámbito de la discapacidad, aquellas empresas de 50 o más trabajadores que no cumplan el requisito de que "al menos el 2 por ciento de sus empleados sean trabajadores con discapacidad", de conformidad con el artículo 42 del Real Decreto Legislativo 1/2013, de 29 de noviembre, por el que se aprueba el texto refundido de la Ley General de derechos de las personas con discapacidad; (2) En el ámbito de la igualdad de género, aquellas empresas de más de 250 trabajadores que no cumplan con la obligación de "contar con un plan de igualdad" conforme a lo dispuesto en el artículo 45 de la Ley Orgánica 3/2007, de 22 de marzo, para la igualdad de mujeres y hombres.

\section{Solvencia técnica social}

En el ámbito medioambiental, según la nueva normativa, se exige determinada experiencia o capacidad para poder seleccionar a las empresas que formarán parte del proceso de adjudicación del contrato. Esta experiencia deberá acreditarse con la presentación de certificados de gestión medioambiental a las empresas licitadoras, como condición de solvencia técnica.

Igualmente, "si el objeto contractual requiriese aptitudes específicas en materia social, de prestación de servicios de proximidad u otras análogas, en todo caso se exigirá como requisito de solvencia técnica o profesional la concreta experiencia, conocimientos y medios en las referidas materias, lo que deberá acreditarse por los medios que establece el apartado 1 de este artículo" (Artículo 90.3). 


\section{Contratos reservados}

La disposición adicional cuarta de la nueva Ley de Contratos establece la posibilidad de reservar el "derecho a participar en los procedimientos de adjudicación de determinados contratos a Centros Especiales de Empleo de iniciativa social y a Empresas de Inserción".

\section{Fase de adjudicación (criterios)}

\section{Criterios de adjudicación}

En esta fase se valorarán las ofertas presentadas por las empresas, una vez que se ha realizado la admisión de las empresas que participarán en la licitación. Hasta ahora primaba el precio ante otros factores, esto es, la oferta económica más ventajosa. En cambio, en la nueva normativa prevalece el criterio de "la mejor relación calidad/precio". Esta medida pretende evitar que la elección de las ofertas con precio más bajo afecte a la calidad, de tal forma que, en la nueva Ley se fomenta la adjudicación de los contratos utilizando criterios de adjudicación en base a la mejor relación calidad-precio. Esto implica que la adjudicación se realice con arreglo a criterios económicos y cualitativos, y, en estos últimos, se podrán incluir aspectos sociales y medioambientales, siempre que estén vinculados al objeto del contrato.

\section{Criterios de preferencia}

Una vez aplicados los criterios de adjudicación, si se produce un empate entre dos o más ofertas de empresas, la nueva Ley establece criterios específicos que se podrán utilizar para el desempate, siempre que estén vinculados al objeto del contrato. En caso de no inclusión de tales criterios, el empate se resolverá atendiendo a "criterios sociales referidos al momento de finalizar el plazo de presentación de ofertas: a) Mayor porcentaje de trabajadores con discapacidad o en situación de exclusión social en la plantilla de cada una de las empresas, primando en caso de igualdad, el mayor número de trabajadores fijos con discapacidad en plantilla, o el mayor número de personas trabajadoras en inclusión en la plantilla; b) Menor porcentaje de contratos temporales en la plantilla de cada una de las empresas; c) Mayor porcentaje de mujeres empleadas en la plantilla de cada una de las empresas; $d$ ) El sorteo, en caso de que la aplicación de los anteriores criterios no hubiera dado lugar a desempate".

\section{Fase de ejecución (condiciones)}

Una vez adjudicado el contrato, llega el momento de su ejecución, donde hasta ahora, la legislación de contratos públicos permitía la inclusión de criterios sociales y medioambientales, pero no obli- 
gaba a ello, por lo tanto, su incorporación a los pliegos era facultativa y no imperativa, por lo que depende de la voluntad del órgano adjudicador el incluir o no dichas cláusulas. A partir de ahora, la Ley impone la obligación al órgano de contratación de establecer en el pliego al menos una de las condiciones especiales de ejecución de tipo medioambiental, social o relativas al empleo que se establecen en el artículo 202 de la citada Ley, siendo imprescindible haberlo establecido previamente en los criterios de adjudicación y siempre que estén vinculadas al objeto del contrato.

\section{Resultado: propuesta de indicadores de medición}

La primera etapa de la investigación reveló la existencia de numerosas guías y publicaciones para la implementación de cláusulas sociales, éticas y medioambientales en las contrataciones públicas, así como de instrucciones o acuerdos sobre contratación pública sostenible. Concretamente, en España, son muchas las administraciones que, en los últimos años, han publicado guías de buenas prácticas para la inclusión de cláusulas de RS en sus contrataciones. La Tabla 1 muestra una relación de cada una de ellas.

\section{Tabla 1. Ejemplos de buenas prácticas sobre contratación pública sostenible en España}

\begin{tabular}{|c|c|c|}
\hline AÑO & ADMINISTRACIÓN & GUÍAS DE BUENAS PRÁCTICAS \\
\hline 2009 & CÓRDOBA & $\begin{array}{l}\text { Guía Práctica para el desarrollo de la contratación pública Sostenible. Diputación de } \\
\text { Córdoba. IDEAS y Asociación Bakeaz. }\end{array}$ \\
\hline 2010 & CASTILLA-LA MANCHA & $\begin{array}{l}\text { Criterios sociales y medioambientales en la contratación pública. Federación Municipios } \\
\text { y Concejos Castilla-La Mancha. María Teresa Brunete de la Llave. }\end{array}$ \\
\hline 2011 & ANDALUCÍA & $\begin{array}{l}\text { Cláusulas Sociales. Dossier para la contratación pública sostenible. Junta de Andalucía. } \\
\text { Fundación para la Atención e Incorporación Social. }\end{array}$ \\
\hline 2011 & GRANADA & $\begin{array}{l}\text { Guía práctica para la incorporación de cláusulas sociales en la contratación pública. } \\
\text { Diputación de Granada. Santiago Lesmes Zabalegui. }\end{array}$ \\
\hline 2011 & MALLORCA & $\begin{array}{l}\text { Guia de clàusules socials. Consell Insular de Mallorca. Departament de Cooperació } \\
\text { Local i Interior Observatori de Polítiques Locals. Santiago Lesmes Zabalegui. }\end{array}$ \\
\hline 2013 & ANDALUCÍA & $\begin{array}{l}\text { Incorporación de cláusulas de igualdad en contratos, subvenciones y convenios públi- } \\
\text { cos. Instituto Andaluz de la Mujer. }\end{array}$ \\
\hline 2016 & GOBIERNO DE ESPAÑA & $\begin{array}{l}\text { Estudio sobre "El uso estratégico de la contratación pública en la promoción de políti- } \\
\text { cas verdes, sociales y de innovación". El caso de España. }\end{array}$ \\
\hline 2016 & VALENCIA & $\begin{array}{l}\text { Guía práctica para la inclusión de cláusulas de Responsabilidad Social en la contrata- } \\
\text { ción y en subvenciones de la Generalitat Valenciana y su sector público. Conselleria de } \\
\text { Transparència, Responsabilitat Social, Participació i Cooperació. }\end{array}$ \\
\hline
\end{tabular}


Por otro lado, se ha observado el compromiso existente de las AAPP españolas con la RS, mediante la aprobación de Acuerdos o Instrucciones. Efectivamente, desde el año 2005, en el que se aprobó en España el Código de Buen Gobierno de la Administración General del Estado y, la Federación Española de Municipios y Provincias (FEMP) aprobó en el año 2009 su propio Código de Buen Gobierno, se han dado pasos muy significativos en materia de RS y sostenibilidad, como se puede apreciar en la Tabla 2.

\section{Tabla 2. Instrucciones o Acuerdos sobre contratación pública sostenible en España}

\begin{tabular}{|c|c|c|}
\hline AÑO & ADMINISTRACIÓN & INSTRUCCIONES Y ACUERDOS \\
\hline 2008 & PAÍS VASCO & $\begin{array}{l}\text { Acuerdo del Gobierno Vasco sobre incorporación de criterios sociales, ambientales y } \\
\text { otras políticas públicas en la contratación de la Administración de la Comunidad } \\
\text { Autónoma y de su sector público. }\end{array}$ \\
\hline 2009 & AVILÉS & $\begin{array}{l}\text { Instrucción para la incorporación de criterios sociales en los contratos públicos del } \\
\text { Ayuntamiento de Avilés. }\end{array}$ \\
\hline 2010 & VITORIA & $\begin{array}{l}\text { Instrucción para la Incorporación de cláusulas sociales en la contratación del } \\
\text { Ayuntamiento de Vitoria-Gasteiz. }\end{array}$ \\
\hline 2011 & PAÍS VASCO & $\begin{array}{l}\text { Circular del Rector para la inclusión de criterios Sociales, éticos y ambientales en las } \\
\text { contrataciones de la Universidad del País Vasco UPV/EHU, aprobada mediante } \\
\text { Resolución de } 22 \text { de marzo de } 2011 \text { de la Secretaría de la UPV/EHU. }\end{array}$ \\
\hline 2012 & CASTELLÓN & $\begin{array}{l}\text { Instrucción para la inclusión de criterios sociales en la contratación pública del } \\
\text { Ayuntamiento de Castellón. }\end{array}$ \\
\hline 2012 & PAMPLONA & $\begin{array}{l}\text { Instrucción de Alcaldía sobre eficiencia en la contratación pública municipal del } \\
\text { Ayuntamiento de Pamplona. }\end{array}$ \\
\hline 2013 & ASTURIAS & $\begin{array}{l}\text { Acuerdo de } 29 \text { de mayo de } 2013 \text {, del Consejo de Gobierno, por el que se aprueban } \\
\text { modelos de cláusulas sociales en el ámbito de la contratación pública. }\end{array}$ \\
\hline 2013 & BARCELONA & $\begin{array}{l}\text { Medida de gobierno para la contratación pública responsable con criterios sociales y } \\
\text { ambientales del Ayuntamiento de Barcelona, de } 15 \text { de marzo de } 2013 \text {. }\end{array}$ \\
\hline 2013 & GUIPÚZCOA & $\begin{array}{l}\text { Norma Foral } 4 / 2013 \text {, de } 17 \text { de julio, de incorporación de cláusulas sociales en los con- } \\
\text { tratos de obras del Sector Público Foral. }\end{array}$ \\
\hline 2013 & VIZCAYA & $\begin{array}{l}\text { Instrucción por la que se establecen criterios sobre mantenimiento de condiciones de } \\
\text { trabajo y medidas de carácter social para su aplicación en los procedimientos de con- } \\
\text { tratación de la Diputación Foral de Bizkaia. }\end{array}$ \\
\hline 2014 & CASTILLA-LA MANCHA & $\begin{array}{l}\text { Ley } 7 / 2014 \text {, de } 13 \text { de noviembre, de Garantía de los Derechos de las Personas con } \\
\text { Discapacidad en Castilla-La Mancha. }\end{array}$ \\
\hline 2014 & EXTREMADURA & $\begin{array}{l}\text { Acuerdo de Consejo de Gobierno por el que se aprueban las directrices de política } \\
\text { general dirigidas a los órganos de contratación de la Administración de la Comunidad } \\
\text { Autónoma de Extremadura sobre contratos reservados e incorporación de cláusulas } \\
\text { sociales en la contratación pública. }\end{array}$ \\
\hline
\end{tabular}




\begin{tabular}{|c|c|c|}
\hline AÑO & ADMINISTRACIÓN & INSTRUCCIONES Y ACUERDOS \\
\hline 2014 & GOBIERNO DE ESPAÑA & Plan de Acción de la Estrategia Española sobre discapacidad 2014-2020. \\
\hline 2014 & GUIPÚZCOA & $\begin{array}{l}\text { Norma Foral } 11 / 2014 \text {, de } 29 \text { de octubre, de la incorporación de cláusulas contractuales } \\
\text { relativas a la compra pública socialmente responsable en la contratación del Sector } \\
\text { Público Foral. }\end{array}$ \\
\hline 2015 & VALENCIA & $\begin{array}{l}\text { Acuerdo de } 27 \text { de marzo de } 2015 \text {, del Consell, por el que se establecen directrices para } \\
\text { la aplicación de cláusulas de carácter social en la contratación de la Administración } \\
\text { de la Generalitat y su sector público, así como en materia de subvenciones de la } \\
\text { Administración de la Generalitat. }\end{array}$ \\
\hline 2015 & VALLADOLID & $\begin{array}{l}\text { Instrucción } 1 / 2015 \text {, de Secretaría General, para impulsar la Eficiencia y Sostenibilidad } \\
\text { en la contratación pública del Ayuntamiento e implementar a través de ella las políticas } \\
\text { municipales en materia social, medioambiental, de innovación y promoción de las } \\
\text { PYMES. }\end{array}$ \\
\hline 2015 & GIJÓN & $\begin{array}{l}\text { Instrucción para la implantación de la contratación pública sostenible en el Ayuntamiento } \\
\text { de Gijón / Xixón y sus organismos autónomos. }\end{array}$ \\
\hline 2016 & ASTURIAS & $\begin{array}{l}\text { Acuerdo de } 10 \text { de febrero de 2016, del Consejo de Gobierno, por el que se reserva el } \\
\text { derecho a participar en los procedimientos de adjudicación de determinados contra- } \\
\text { tos a Centros Especiales de Empleo y a empresas de inserción, y se establecen los } \\
\text { porcentajes mínimos de esta reserva y las condiciones mínimas para garantizar su cum- } \\
\text { plimiento. }\end{array}$ \\
\hline 2016 & CASTILLA Y LEÓN & $\begin{array}{l}\text { Acuerdo 44/2016, de } 21 \text { de julio, de la Junta de Castilla y León, por el que se aprueban } \\
\text { directrices vinculantes para los órganos de contratación de la Administración de la } \\
\text { Comunidad de Castilla y León sobre incorporación de cláusulas sociales en la contra- } \\
\text { tación pública. }\end{array}$ \\
\hline 2016 & ISLAS BALEARES & $\begin{array}{l}\text { Acuerdo del Consejo de Gobierno de } 29 \text { de abril de } 2016 \text {, por el que se establecen direc- } \\
\text { trices para la inclusión de cláusulas de carácter social en la contratación de la } \\
\text { Administración de la Comunidad Autónoma de las Illes Balears y su sector público ins- } \\
\text { trumental. }\end{array}$ \\
\hline 2016 & MADRID & $\begin{array}{l}\text { Acuerdo de } 4 \text { de febrero de } 2016 \text { de la Junta de Gobierno de la Ciudad de Madrid por } \\
\text { el que se establecen directrices para la aplicación de la reserva del derecho a partici- } \\
\text { par en la contratación municipal a los centros especiales de empleo y empresas de } \\
\text { inserción y se fija el porcentaje mínimo de participación para } 2016 \text {. } \\
\text { Decreto de } 19 \text { de enero de } 2016 \text {, del Delegado del Área de Gobierno de Economía y } \\
\text { Hacienda, por el que se aprueba la Instrucción 1/2016, relativa a la incorporación de } \\
\text { cláusulas sociales en los contratos celebrados por el Ayuntamiento de Madrid, sus orga- } \\
\text { nismos autónomos y entidades del sector público municipal. }\end{array}$ \\
\hline
\end{tabular}

En la segunda etapa del estudio se procedió a la creación de una serie de indicadores sociales y ambientales que sirvan para poder evaluar y comparar las prácticas responsables en las contrataciones públicas, que hemos dividido en las distintas fases del contrato (Tabla 3). 


\section{Tabla 3. Indicadores por fases}

\begin{tabular}{|l|c|c|}
\hline Fases del contrato & $\mathbf{N}^{\circ}$ de indicadores sociales & N de indicadores medioambientales \\
\hline Fase de preparación & 5 & 2 \\
Fase de adjudicación & 11 & 10 \\
Fase de ejecución & 10 & 10 \\
\hline Total indicadores & 26 & $\mathbf{2 2}$ \\
\hline
\end{tabular}

En las Tablas 4, 5, 6, 7 y 8, se recogen los indicadores propuestos, desglosados por fases del contrato, como se ha mencionado anteriormente, y por dimensiones, social y medioambiental.

\section{Tabla 4. Propuesta de indicadores sociales fase de preparación del contrato}

\begin{tabular}{|l|l|}
\hline Fase de preparación & Indicadores \\
\hline Objeto del contrato & $\begin{array}{l}\text { Alusiones sociales explícitas en la definición del objeto del contrato } \\
\text { Cumplimiento de la obligación de contratar un 2\% de personas con discapacidad (50 o más } \\
\text { trabajadores) } \\
\text { Cumplimiento de la obligación de contar con un Plan de Igualdad } \\
\text { Experiencia en prestación de servicios sociales } \\
\text { Solvencia Técnica social } \\
\text { Contratos reservados }\end{array}$ \\
$\begin{array}{l}\text { Reserva la participación en la licitación a Centros Especiales de Empleo y Empresas de } \\
\text { Inserción y otras Entidades destinadas a la Inserción con una discapacidad que, debido a } \\
\text { la indole o a la gravedad de sus deficiencias, no puedan ejercer una actividad profesional } \\
\text { en condiciones normales, y/o personas en riesgo de exclusión social legalmente constitui- } \\
\text { dos y registrados }\end{array}$ \\
\hline
\end{tabular}




\section{Tabla 5. Propuesta de indicadores sociales fase de adjudicación del contrato}

\begin{tabular}{|l|l|}
\hline Fase de adjudicación & Indicadores \\
\hline Criterios de adjudicación & $\begin{array}{l}\text { Puntuación por contratación de personas con discapacidad } \\
\text { Puntuación por contratación de personas o colectivos en situación o riesgo de exclusión } \\
\text { social } \\
\text { Puntuación por utilización de productos de Comercio Justo } \\
\text { Puntuación por contratación de mujeres } \\
\text { Puntuación por establecer Planes de igualdad y/o medidas de conciliación } \\
\text { Puntuación por mejorar las condiciones laborales } \\
\text { Puntuación por generación de empleo } \\
\text { Puntuación por subcontratación de Empresas de Inserción y Centros Especiales de Empleo }\end{array}$ \\
\hline $\begin{array}{l}\text { Criterios de preferencia } \\
\text { (empate) }\end{array}$ & $\begin{array}{l}\text { Mayor porcentaje de trabajadores con discapacidad en plantilla } \\
\text { Menor porcentaje de trabajadores temporales en plantilla } \\
\text { Mayor porcentaje de mujeres en plantilla }\end{array}$ \\
\hline
\end{tabular}

\section{Tabla 6. Propuesta de indicadores sociales fase de ejecución del contrato}

\begin{tabular}{|l|l|}
\hline Fase de ejecución & Indicadores sociales \\
\hline Condiciones de ejecución & $\begin{array}{l}\text { Obligación de contratar personas con discapacidad } \\
\text { Obligación de emplear a personas en situación o riesgo de exclusión social } \\
\text { Obligación de utilizar productos de Comercio Justo } \\
\text { Obligaciones en materia de igualdad de mujeres y hombres } \\
\text { Obligar el cumplimiento a lo largo de toda la ejecución contractual de todas las disposicio- } \\
\text { nes legales, reglamentarias y convencionales en vigor en materia laboral, de seguridad social, } \\
\text { y de seguridad y salud en el trabajo que sean aplicables a las personas trabajadoras vin- } \\
\text { culadas a la ejecución del contrato } \\
\text { Obligar a emplear un porcentaje mínimo de plantilla indefinida sobre el total del personal } \\
\text { Obligación de mantener el empleo y la plantilla } \\
\text { Obligación de cumplir derechos humanos y criterios éticos } \\
\text { Obligación de no tributar en paraísos fiscales } \\
\text { Obligación de contratar con Empresas de Inserción y Centros Especiales de Empleo }\end{array}$ \\
\hline
\end{tabular}




\title{
Tabla 7. Propuesta de indicadores medioambientales fase de preparación del contrato
}

\begin{tabular}{|l|l|}
\hline Fase de preparación & Indicadores \\
\hline $\begin{array}{l}\text { Objeto del contrato } \\
\text { Solvencia Técnica social }\end{array}$ & $\begin{array}{l}\text { Alusiones medioambientales explícitas en la definición del objeto del contrato } \\
\text { Certificados de gestión medioambiental }\end{array}$ \\
\hline
\end{tabular}

\section{Tabla 8. Propuesta de indicadores medioambientales fase de adjudicación y ejecución del contrato}

\author{
Indicadores \\ - Reducción de las emisiones de gases de efecto invernadero, contribuyendo así a dar cumplimiento al obje- \\ tivo que establece el artículo 88 de la Ley 2/2011, de 4 de marzo, de Economía Sostenible \\ - Empleo de medidas de ahorro y eficiencia energética \\ - Utilización de energía procedentes de fuentes renovables durante la ejecución del contrato \\ - Utilización de productos ecológicos \\ - Mantenimiento o mejora de los recursos naturales que puedan verse afectados por la ejecución del contrato \\ - Gestión más sostenible del agua \\ - Fomento del uso de las energías renovables \\ - Promoción del reciclado de productos y el uso de envases reutilizables \\ - Impulso de la entrega de productos a granel y la producción ecológica
}

\section{Conclusiones}

De acuerdo a los objetivos que nos hemos planteado en este estudio exploratorio, hemos comprobado que, cada vez más y con carácter general, las AAPP son conscientes y se preocupan por los aspectos que tienen que ver con la Transparencia, la RS y la sostenibilidad. Del análisis de la situación actual de las contrataciones públicas sostenibles, constatamos los importantes esfuerzos de las iniciativas, tanto nacionales como internacionales, encaminadas a fortalecer un marco regulatorio en 
esta materia. Concretamente, en España, se observa que son muchas las administraciones que adoptan el compromiso de ser socialmente responsables en sus contrataciones. Sin embargo, hemos podido comprobar la gran tarea que queda por hacer para adaptarse al marco normativo en estas materias.

Según nuestros conocimientos, este es el primer estudio en el que se han definido una serie de indicadores que sirven para evaluar la sostenibilidad en las contrataciones públicas, de ahí su importancia. Los indicadores que proponemos, básicamente permiten orientar la implementación de buenas prácticas y pueden ser utilizados en investigaciones como un instrumento metodológico para seguir progresando en la comprensión y mejora de estos procesos de contratación pública sostenible.

Como todo estudio exploratorio, además de servir de base para futuras investigaciones, constituye un elemento generador de hipótesis. En este sentido a las preguntas de investigación antes mencionadas y nuestra propuesta de medida servirá para poder responderlas con la suficiente confianza, utilidad y validez cuando obtengamos datos cuantitativos, dado que ahora no disponemos de información.

Señalar, también, la necesidad de un estudio más profundo, que permita evaluar los comportamientos responsables de las AAPP en relación a sus contrataciones, así como observar la evolución en el tiempo, poder efectuar comparaciones y disponer de una visión global del estado de la RS en las contrataciones del sector público. Para ello, proponemos un análisis de contenido de los pliegos de condiciones de las AAPP, utilizando los indicadores propuestos, asignándoles valores para poder cuantificarlos. Planteamos, por tanto, la construcción de índices que nos permitan cuantificar el grado de comportamiento responsable y, así, poder establecer rankings y comparaciones entre las administraciones. Asimismo, podemos establecer modelos de relaciones causales con el objetivo de poder determinar cuáles son los factores que condicionan la sostenibilidad en las contrataciones públicas.

Todo ello permitirá conocer, además de la situación actual de las contrataciones públicas sostenibles y poder compararlas en el tiempo, cuál ha sido el impacto de la aprobación de las leyes de contratación en las administraciones locales, de las buenas prácticas en la contratación pública; cuáles son los efectos de las instrucciones o acuerdos sobre contratación pública; si existen patrones de comportamiento entre las AAPP en relación a sus contrataciones públicas responsables 0 cuáles podrían ser los factores condicionantes de las prácticas sostenibles en las contrataciones públicas. 


\section{Referencias bibliográficas}

ALDEGUER, B. (2013): "Ética Pública y Gobierno Local en un contexto de crisis económica”, Barataria, 15, $15-37$.

BRAMMER, S. \& WALKER, H. (2011): "Sustainable procurement in the public sector: an international comparative study", International Journal of Operations \& Production Management, 31(4), $452-$ 476. DOI: https://doi.org/10.1108/014435711111119551.

BRUGUÉ, J. (2004): "Modernizar la administración desde la izquierda: burocracia, nueva gestión pública y administración deliberativa", Revista del CLAD Reforma y Democracia, 29, 1-16.

BURZACO, S.M. (2016). "Contrataciones públicas socialmente responsables: la necesidad de reconsiderar el potencial de la contratación pública en la consecución de objetivos sociales", CIRIECEspaña, Revista de Economía Pública, Social y Cooperativa, 86, 281-310.

CANALES, J.M. (2003): "Algunas reflexiones sobre los principales problemas y desafíos pendientes en la innovación de las Administraciones Publicas". En: Fernández, M., Sierra, J. y Valero, J. (coords.), Nuevos Retos en el Horizonte de las Administraciones Públicas, Facultad de Derecho de la Universidad de Murcia. Disponible en: http://www.um.es/gap/nuevos_retos_en_el_horizonte_de_las_AAPP.pdf.

CUETO, C. (2013): Responsabilidad Social en la AP y modelo europeo de excelencia en la gestión (EFQM), UNED, Cátedra de RS y Sostenibilidad.

GARCÍA, J.B. (2013): "Clásulas sociales en la contratación pública: nuevos instrumentos para el fomento del empleo a nivel local/Social clauses in public procurement: new tools to promote local employment", Cuadernos de Trabajo Social, 26(1), 85.

DOI: http://dx.doi.org/10.5209/rev_CUTS.2013.v26.n1.40691

GELDERMAN, C.J., SEMEIJN, J. \& VLUGGEN, R. (2017): "Development of sustainability in public sector procurement", Public Money \& Management, 37(6), 435-442.

DOl: https://doi.org/10.1080/09540962.2017.1344027.

GUTIÉRREZ-PONCE, H., CANO-MONTERO, E. \& CHAMIZO-GONZÁLEZ, J. (2018): "Estabilidad presupuestaria, financiación y responsabilidad social en los municipios españoles", Contaduría y Administración, 63(3), 36-37. DOI: http://dx.doi.org/10.22201/fca.24488410e.2018.1419.

HERNÁNDEZ, R., FERNÁNDEZ, C. \& BAPTISTA, P. (2003): Metodología de la investigación (3ª ed.), México: McGraw-Hill. 
JIMÉNEZ, R. (2013): "Ética Pública, política y alta administración: Los Códigos Éticos como vía para reforzar el Buen Gobierno", Revista Vasca de Gestión de Personas y Organizaciones Públicas, 5, 46-67.

MELLE, M. (2007): "La responsabilidad social dentro del sector público", Ekonomiaz, 65, 84-107.

NEVADO, M., GALLARDO, D. \& SÁNCHEZ, M. (2013): "La Administración Local y su implicación en la creación de una cultura socialmente responsable", Prisma Social, 10, 64-118.

ORUEZABALA, G. \& RICO, J.C. (2012): "The impact of sustainable public procurement on supplier management-The case of French public hospitals", Industrial Marketing Management, 41(4), 573580. DOI: https://doi.org/10.1016/j.indmarman.2012.04.004.

ROMODINA, I. \& SILIN, M. (2016): "Perspectives of Introduction Sustainable Procurement in Public Procurement in Russia", Oeconomia Copernicana, 7(1), 35-48.

DOI: http://dx.doi.org/10.12775/OeC.2016.003.

SÁNCHEZ, J.J. (2001): La administración pública como ciencia. Su objeto y estudio, México: Plaza y Valdés, S.A.

SHADRINA, E. \& ROMODINA, I. (2017): "Sustainable Public Procurement: International Experience", Public administration issues, 1, 149-172.

SUBIRATS, J. (2012): “¿Qué democracia y qué Administración para la nueva época? Internet y la gestión pública", Ekonomiaz, 80 (2), 70-91.

SUBIRATS, J. (2003): Análisis de Políticas Públicas y eficacia de la Administración, Madrid: INAP.

TRUJILLO, E., LÓPEZ, M.J. \& ÁLVAREZ, O. (2011): Manual de Gestión Pública Responsable, Madrid: Fundación Internacional y para Iberoamérica de Administración y Políticas.

VAÑÓ, M.J. (2016): "Limitaciones a la aplicación de cláusulas sociales en la contratación pública desde la perspectiva del derecho de la competencia", CIRIEC-España, Revista de Economía Pública, Social y Cooperativa, 87, 177-202.

VILLORIA, M. (2006): La corrupción política, Madrid: Síntesis. 


\section{LEGISLACIÓN, ACUERDOS E INSTRUCCIONES}

ANDALUCÍA (2011): Cláusulas Sociales. Dossier para la contratación pública sostenible. Junta de Andalucía. Fundación para la Atención e Incorporación Social. Disponible en: http://www.conr.es/sites/defaultfiles/archivos/Clausulas\%20Sociales.\%20Dossier\%20para\%201a\%20con tratacion\%20publica\%20sostenible.\%20Junta\%20de\%20Andalucia..pdf. Acceso: 2 de diciembre de 2017.

ANDALUCÍA (2013): Incorporación de cláusulas de igualdad en contratos, subvenciones y convenios públicos. Instituto Andaluz de la Mujer. Disponible en: http://www.juntadeandalucia.es/turismoydeporte/export/sites/ctc/.galleries/descargas-unidad-genero/Guia_contratacion_web.pdf. Acceso: 2 de enero de 2018.

ASTURIAS (2013): Acuerdo de 29 de mayo de 2013, del Consejo de Gobierno, por el que se aprueban modelos de cláusulas sociales en el ámbito de la contratación pública, Boletín Oficial del Principado de Asturias, 130, 1-2. Disponible en: https://sede.asturias.es/bopa/2013/06/06/201310601.pdf Acceso: 3 de marzo de 2018.

ASTURIAS (2016): Acuerdo de 10 de febrero de 2016, del Consejo de Gobierno, por el que se reserva el derecho a participar en los procedimientos de adjudicación de determinados contratos a Centros Especiales de Empleo y a empresas de inserción, y se establecen los porcentajes mínimos de esta reserva y las condiciones mínimas para garantizar su cumplimiento, Boletín Oficial del Principado de Asturias, 39, 1-3. Disponible en: https://sede.asturias.es/bopa/2016/02/17/201601531.pdf. Acceso: 10 de marzo de 2018.

AVILÉS (2009): Instrucción para la inclusión de criterios sociales en la contratación pública del Ayuntamiento de Avilés. Boletín Oficial del Principado de Asturias, 273, 1-10. Disponible en: https://sede.asturias.es/bopa/2009/11/25/2009-26730.pdf. Acceso: 10 de marzo de 2018.

BARCELONA (2013): Medida de gobierno para la contratación pública responsable con criterios sociales y ambientales del Ayuntamiento de Barcelona, de 15 de marzo de 2013. Ajuntament de Barcelona. Disponible en: https://www.uv.es/empresocial/Seminaris/Jornada_lbercaja/ponencias/05-Decreto_clausulas_sociales.pdf. Acceso: 2 de mayo de 2018.

CASTELLÓN (2012): Instrucción para la inclusión de criterios sociales en la contratación pública del Ayuntamiento de Castellón. Boletín Oficial de la Provincia de Castellón. Disponible en: http://www.castello.es/archivos/1169/Anuncio_BOP_Instruccion.pdf. Acceso: 3 de mayo de 2018

CASTILLA-LA MANCHA (2010): Criterios sociales y medioambientales en la contratación pública. Federación Municipios y Concejos Castilla la Mancha. Federación de Municipios y Provincias Castilla-La Mancha. Disponible en:

http://www.conr.es/sites/default/files/archivos/Criterios\%20sociales\%20y\%20medioambienta les\%20en\%20la\%20contratacion\%20publica.\%20Federacion\%20Municipios\%20y\%20Provincias\%20C LM.pdf. Acceso: 13 de septiembre de 2017. 
CASTILLA-LA MANCHA (2014): Ley 7/2014, de 13 de noviembre, de Garantía de los Derechos de las Personas con Discapacidad en Castilla-La Mancha. Diario Oficial de Castilla-La Mancha, 233, 37591-37619. Disponible en:

http://docm.jccm.es/portaldocm/descargarArchivo.do?ruta=2014/12/02/pdf/2014_15009.pdf\&tipo=rutaDo cm. Acceso: 14 de mayo de 2018.

CASTILLA Y LEÓN (2016): Acuerdo 44/2016, de 21 de julio, de la Junta de Castilla y León, por el que se aprueban directrices vinculantes para los órganos de contratación de la Administración de la Comunidad de Castilla y León sobre incorporación de cláusulas sociales en la contratación pública. Boletín Oficial de Castilla y León, 142, 34770-34799. Disponible en:

http://bocyl.jcyl.es/boletines/2016/07/25/pdf/BOCYL-D-25072016-10.pdf. Acceso: 18 de enero de 2018.

COMISIÓN EUROPEA (COM) (2001): Libro Verde. Fomentar un marco europeo para la responsabilidad social de las empresas. Comisión Europea, Bruselas. 366-final. Disponible en: http://www.europarl.europa.eu/meetdocs/committees/deve/20020122/com(2001)366_es.pdf. Acceso: 8 de enero de 2018.

COMISIÓN EUROPEA (COM) (2008): Communication from the commission to the European Parliament, the council, the European Economic and Social Committee and the Committee of Regions. Public procurement for a better environment, Bruselas. Disponible en:

http://www.europarl.europa.eu/meetdocs/2009_2014/documents/com/com_com(2011)0681_/com_com( 2011)0681_en.pdf. Acceso: 18 de enero de 2018.

COMISIÓN EUROPEA (COM) (2011): Adquisiciones sociales: Una guía para considerar aspectos sociales en las públicas. Disponible en:

http://www.prodetur.es/prodetur/AlfrescoFileTransferServlet?action=download\&ref=dafd3637-7c4547e6-bbb0-cce13b4726d3. Acceso: 31 de enero de 2018.

CÓRDOBA (2009): Guía Práctica para el desarrollo de la contratación pública Sostenible. Diputación de Córdoba. IDEAS y Asociación Bakeaz. Diputación de Córdoba. Disponible en: http://www.conr.es/sites/default/files/archivos/Guia\%20practica\%20para\%20el\%20desarro $110 \% 20$ de\%20la\%20contratacion\%20publica\%20sostenible.\%20Diputacion\%20Cordoba..pdf. Acceso: 3 de noviembre de 2017.

ESPAÑA (2006): Ley 39/2006, de 14 de diciembre, de Promoción de la Autonomía Personal y Atención a las personas en situación de dependencia. Boletín Oficial del Estado, 299, 44142-44156.

ESPAÑA (2007): Ley Orgánica 3/2007, de 22 de marzo, para la igualdad efectiva de mujeres y hombres. Boletín Oficial del Estado, 71, 12611-12645.

ESPAÑA (2007): Ley 26/2007, de 23 de octubre, de Responsabilidad Medioambiental. Boletín Oficial del Estado, 255, 43229-43250.

ESPAÑA (2007): Ley 45/2007, de 13 de diciembre, para el desarrollo sostenible del medio rural. Boletín Oficial del Estado, 299, 51339-51349. 
ESPAÑA (2011): Ley 2/2011, de 4 de marzo de Economía Sostenible. Boletín Oficial del Estado, 55, 25033-25235.

ESPAÑA (2013): Ley 19/2013, de 9 de diciembre, de Transparencia, Acceso a la Información Pública y Buen Gobierno. Boletín Oficial del Estado, 295, 97922-97952.

ESPAÑA (2013): Real Decreto Legislativo 1/2013, de 29 de noviembre, por el que se aprueba el Texto Refundido de la Ley General de derechos de las personas con discapacidad y de su inclusión social. Boletín Oficial del Estado, 289, 95635-95672.

ESPAÑA (2016): Estudio sobre "El uso estratégico de la contratación pública en la promoción de políticas verdes, sociales y de innovación". El caso de España. Disponible en:

http://www.conr.es/sites/default/files/archivos/ES\%20Country\%20Report_v3-0.pdf. Acceso: 13 de marzo de 2018.

ESPAÑA (2017): Ley 9/2017, de 8 de noviembre, de Contratos del Sector Público, por la que se transponen al ordenamiento jurídico español las Directivas del Parlamento Europeo y del Consejo 2014/23/UE y 2014/24/UE, de 26 de febrero de 2014. Boletín Oficial del Estado, 272, 107714108007.

EXTREMADURA (2014): Resolución de 1 de agosto de 2014, de la Consejera, por la que se dispone la publicación del Acuerdo de Consejo de Gobierno de 8 de julio de 2014, por el que se aprueban las directrices de política general dirigidas a los órganos de contratación de la Administración de la Comunidad Autónoma de Extremadura sobre contratos reservados e incorporación de cláusulas sociales en la contratación pública. Diario Oficial de Extremadura, 150, 24372-24383. Disponible en: http://doe.juntaex.es/pdfs/doe/2014/1500o/14061610.pdf. Acceso: 3 de marzo de 2018.

GIJÓN (2015): Instrucción para la implantación de la contratación pública sostenible en el Ayuntamiento de Gijón / Xixón y sus organismos autónomos. Disponible en: https://sedeelectronica.gijon.es/multimedia_objects/download?object_id=190369\&object_type=document. Acceso: 23 de marzo de 2018.

GOBIERNO DE ESPAÑA (2014): Plan de Acción de la Estrategia Española sobre Discapacidad 20142020. Ministerio de Sanidad, Servicios Sociales e Igualdad. Disponible en: https://www.msssi.gob.es/ssi/discapacidad/docs/plan_accion_EED.pdf. Acceso: 14 de marzo de 2018.

GRANADA (2011): Guía práctica para la incorporación de cláusulas sociales en la contratación pública. Diputación de Granada. Santiago Lesmes Zabalegui. Disponible en: http://www.reasnet.com/clausulassociales/documentos/guias/Gu\%C3\%ADa\%20CCSS\%20Diputaci\%C3\%B3n\%20Granada.pdf Acceso: 12 de noviembre de 2017.

GUIPÚZCOA (2013): Norma Foral 4/2013, de 17 de julio, de incorporación de cláusulas sociales en los contratos de obras del Sector Público Foral. Boletín Oficial del País Vasco, 182. Disponible en: http://www4.gipuzkoa.net/ogasuna/normativa/docs/0000976c.pdf. Acceso: 15 de marzo de 2018. 
GUIPÚZCOA (2014): Norma Foral 11/2014, de 29 de octubre, de incorporación de cláusulas contractuales relativas a la compra pública socialmente responsable en la contratación del sector público foral, Boletín Oficial de Gipuzkoa, 207. Disponible en:

https://egoitza.gipuzkoa.eus/ogasuna/normativa/docs/0001016c.pdf. Acceso: 23 de marzo de 2018.

ISLAS BALEARES (2016): Acuerdo del Consejo de Gobierno de 29 de abril de 2016, por el que se establecen directrices para la inclusión de cláusulas de carácter social en la contratación de la Administración de la Comunidad Autónoma de las Illes Balears y su sector público instrumental. Butlletí Oficial de les Illes Balears, Disponible en:

https://s3-eu-west-1.amazonaws.com/codekstudio-dpp/guias/Acuerdo+Gobierno+Baleares.pdf Acceso: 24 de abril de 2018.

MADRID (2016): Acuerdo de 4 de febrero de 2016 de la Junta de Gobierno de la Ciudad de Madrid por el que se establecen directrices para la aplicación de la reserva del derecho a participar en la contratación municipal a los centros especiales de empleo y empresas de inserción y se fija el porcentaje mínimo de participación para 2016. Boletín Oficial del Ayuntamiento de Madrid, 7593. Disponible en: http://www.conr.es/sites/default/files/archivos/Ayuntamiento\%20de\%20Madrid\%20\%20Acuerdo\%20de\%204\%20de\%2Ofebrero\%20de\%202016\%20sobre\%20Reserva\%20de\%20Contrat os\%20a\%20CEE.pdf. Acceso: 3 de mayo de 2018.

MADRID (2016): Decreto de 19 de enero de 2016, del Delegado del Área de Gobierno de Economía y Hacienda, por el que se aprueba la Instrucción 1/2016, relativa a la incorporación de cláusulas sociales en los contratos celebrados por el Ayuntamiento de Madrid, sus organismos autónomos y entidades del sector público municipal. Disponible en:

http://www.amei.es/wp-content/uploads/2017/09/4.2-Decreto-19-01-16-Clausulas-sociales-Ayto.Madrid.pdf. Acceso: 3 de marzo de 2018.

MALLORCA (2011): Guia de clàusules socials. Consell Insular de Mallorca. Departament de Cooperació Local i Interior Observatori de Polítiques Locals. Santiago Lesmes Zabalegui. Consell de Mallorca. Disponible en:

https://s3-eu-west-1.amazonaws.com/codekstudio-dpp/guias/26.+Guia+de+clausules+socials.pdf Acceso: 3 de marzo de 2018.

MINISTERIO DE TRABAJO E INMIGRACIÓN (MTIN) (2009): Informe sobre la actuación del gobierno en el fomento de la responsabilidad social de las empresas en España. Disponible en: https://www.carm.es/web/integra.servlets.Blob?ARCHIVO=MTIN_Informe\%20actuaci\%F3n\%20Gobiern 0\%20fomento\%20RSE.pdf\&TABLA=ARCHIVOS\&CAMPOCLAVE=IDARCHIVO\&CAMPOIMAGEN=ARCHIVO\&IDTIPO=60\&VALORCLAVE=78642. Acceso: 2 de diciembre de 2017.

PAÍS VASCO (2008): Acuerdo del Gobierno Vasco sobre incorporación de criterios sociales, ambientales y otras políticas públicas en la contratación de la Administración de la Comunidad Autónoma y de su sector público. Boletín Oficial del País Vasco. Disponible en: https://www.euskadi.eus/y22bopv/es/bopv2/datos/2008/06/0803741a.pdf. Acceso: 3 de marzo de 2018. 
PAÍS VASCO (2011): Circular del Rector para la inclusión de criterios Sociales, éticos y ambientales en las contrataciones de la Universidad del País Vasco UPV/EHU, aprobada mediante Resolución de 22 de marzo de 2011 de la Secretaría de la UPV/EHU. Euskampus. Disponible en: https://www.ehu.eus/documents/1180425/1344057/Circular+de+criterios+sociales\%2C\%20\%C3\%A9tico s+y+ambientales.pdf/37eba6b2-c25b-4fb2-a93a-5c1b9f84adbf. Acceso: 14 de marzo de 2018.

PAMPLONA (2012): Instrucción de Alcaldía sobre eficiencia en la contratación pública municipal del Ayuntamiento de Pamplona. Ayuntamiento de Pamplona. Disponible en:

http://www.pamplona.es/verdocumento/verdocumento.aspx?iddoc=241707. Acceso: 16 de marzo de 2018.

VALENCIA (2015): Acuerdo de 27 de marzo de 2015, del Consell, por el que se establecen directrices para la aplicación de cláusulas de carácter social en la contratación de la Administración de la Generalitat y su sector público, así como en materia de subvenciones de la Administración de la Generalitat. Diari Oficial de la Comunitat Valenciana. Disponible en:

https://www.dogv.gva.es/datos/2015/04/02/pdf/2015_2935.pdf. Acceso: 13 de marzo de 2018.

VALENCIA (2016): Guía práctica para la inclusión de cláusulas de Responsabilidad Social en la contratación y en subvenciones de la Generalitat Valenciana y su sector público. Conselleria de Transparència, Responsabilitat Social, Participació i Cooperació. Disponible en: http://www.transparencia.gva.es/documents/162282364/162406386/Gu\%C3\%ADa+de+Cl\%C3\%A1usulas+de+Respon sabilidad+Social/4420cf90-8d86-47b9-9d7c-9cddc4e0dfe9. Acceso: 15 de mayo de 2018.

VALLADOLID (2015): Instrucción 1/2015, de Secretaría General, para impulsar la Eficiencia y Sostenibilidad en la contratación pública del Ayuntamiento e implementar a través de ella las políticas municipales en materia social, medioambiental, de innovación y promoción de las PYMES. Ayuntamiento de Valladolid. Disponible en: http://www.conr.es/sites/default/files/archivos/ NSTRUCCI\%C3\%93N\%20Ayto\%20Valladolid.\%201-2015\%20CONTRATACI\%C3\%93N\%20Estrat\% C3\%A9gica\%20y\%20Transparente.pdf. Acceso: 23 de abril de 2018.

VITORIA (2010): Instrucción para la Incorporación de cláusulas sociales en la contratación del Ayuntamiento de Vitoria-Gasteiz. Disponible en:

https://www.vitoria-gasteiz.org/docs/wb021/contenidosEstaticos/adjuntos/es/30/24/63024.pdf Acceso: 12 de abril de 2018.

VIZCAYA (2013): Instrucción aprobada por acuerdo de consejo de gobierno de la Diputación Foral de Bizkaia de 29 de octubre de 2013 por la que se establecen criterios sobre mantenimiento de condiciones de trabajo y medidas de carácter social para su aplicación en los procedimientos de contratación de la Diputación Foral de Bizkaia. Diputación de Bizkaia. Disponible en:

http://www.bizkaia.eus/lehendakaritza/Bao_bob/2013/11/20131114a218.pdf?hash=20940ba1a813fdc48 cf9fdbb318bc523\#page=6. Acceso: 16 de marzo de 2018. 